\title{
A Prediction Model for Short-Term Neonatal Outcomes in Severe Early-Onset IUGR
}

Andrew Sharp ${ }^{1}$; Christine Cornforth ${ }^{2}$; Richard Jackson²; Jane Harrold ${ }^{2}$; Mark A. Turner ${ }^{1}$; Louise Kenny ${ }^{1}$, Philip N. Baker ${ }^{4}$, Edward D. Johnstone ${ }^{5}$, Asma Khalili ${ }^{6}$, Peter von Dadelszen $^{7}$, Aris T. Papageorghiou ${ }^{6}$, Zarko Alfirevic ${ }^{1}$

\section{Affiliations:}

1. Department of Women's and Children's Health, University of Liverpool, United Kingdom

2. Liverpool Clinical Trials Unit, University of Liverpool, United Kingdom

3. College of Medicine, University of Leicester, United Kingdom

4. Maternal \& Fetal Health Research Centre, University of Manchester, United Kingdom

5. Fetal Medicine Unit, St George's Hospital, and St George's, University of London, United Kingdom

6. Department of Women's and Children's Health, School of Life Course Sciences, King's College London, United Kingdom

\section{Correspondence:}

Professor Zarko Alfirevic: zarko@liverpool.ac.uk

Department of Women's and Children's Health Research, University of Liverpool, Liverpool Women's Hospital, Crown Street, Liverpool, L8 7SS, United Kingdom.

Tel: +441517959550 


\section{BACKGROUND}

Severe early-onset fetal growth restriction (FGR) predisposes to fetal death, neonatal death, neonatal morbidity and neurodisability. The use of placental biomarkers has been proposed for risk stratification in pre-eclampsia but they could be equally useful in FGR in order to aid management.

\section{METHODS}

This is a secondary analysis of the multicenter, placebo-controlled STRIDER trial of singleton pregnancies with severe early-onset FGR. Women with a pregnancy complicated by FGR between $22^{+0}$ and $29^{+6}$ weeks of gestation were randomly assigned to receive either sildenafil $25 \mathrm{mg}$ three times daily or placebo until $32^{+0}$ weeks' gestation or delivery. We developed prediction models for livebirth, gestation at birth, birth weight, overall survival and neonatal morbidity based upon maternal demographics (age, parity, blood pressure, preeclampsia, gestational hypertension), fetal biometric (estimated fetal weight) and Doppler measurements (Middle Cerebral Artery, Umbilical Artery) and maternal angiogenic biomarkers (PIGf, sEng, sFIt-1 and sFIt-1:PIGF ratio).

\section{RESULTS}

A complete data set was available for 105 out 135 randomised women. Multivariable analysis identified estimated fetal weight (EFW) and soluble fms-like tyrosine kinase 1: placental growth factor ratio (sFIt-1:PIGF) as independent predictors of livebirth and overall survival. EFW was a consistent predictor for all outcomes other than gestation at delivery. sFIt-1:PIGF ratio was a consistent predictor for all outcomes other than neonatal morbidity.

\section{CONCLUSIONS}

In severe early-onset FGR pregnancies livebirth and overall survival can be predicted using a model involving EFW and sFIt-1:PIGF ratio. This model will allow informed decision making about pregnancy management, especially in cases that may be considered previable. (Funded by the National Institute of Heath Research and Medical Research Council, ISRCTN39133303) 


\section{Introduction}

Severe early-onset fetal growth restriction (FGR) is associated with significant adverse pregnancy outcomes, which includes; fetal and neonatal death ${ }^{1-3}$, necrotizing enterocolitis ${ }^{3}$, respiratory complications ${ }^{3}$, neurodisability ${ }^{4-7}$ and lifelong risks to the health of the affected child ${ }^{8-10}$. Currently, there is no effective treatment for FGR with women being offered a choice of; 1) expectant management with intensive surveillance and iatrogenic preterm delivery or 2) termination of pregnancy ${ }^{11}$.

We conducted a RCT to test the hypothesis that sildenafil, a phosphodiesterase 5 (PDE5) inhibitor, could prolong gestation improving the blood supply to the placental bed ${ }^{12}$. However, our RCT demonstrated no evidence of benefit in either short term fetal or neonatal outcomes in those women treated with sildenafil ${ }^{13}$. Despite these negative findings the STRIDER RCT does provide valuable clinical and biomarker information for early-onset FGR pregnancies with a more extreme phenotype than other previously published cohorts ${ }^{1,11}$.

To date the ability of risk stratification to predict those fetuses that will go on to be severely growth restricted has been poor ${ }^{14}$. With the addition of detailed biomarker assessment in the first trimester, the risk of fetal growth restriction can be modified but remains imprecise ${ }^{14}$. The mainstay of currently advocated prediction models is based on the pathophysiological similarities between preeclampsia and FGR, both of which share similar patterns of placental dysfunction ${ }^{15}$.

More useful, and distinctly lacking at present, are large studies to give women and clinicians data on which to base a prognosis for the pregnancy, particularly when diagnosis is made at extremely early gestations where viability is uncertain. 
Published FGR cohorts such as the TRUFFLE study provide data on the risk of perinatal mortality and neurological impairment at 2 years of age in pregnancies with moderate to severe FGR ${ }^{7,11}$. The results of this study demonstrated that the risk of fetal demise is very low $(<2 \%)$ when estimated fetal weight is $>500 \mathrm{~g}$, Umbilical artery Doppler is abnormal (raised PI or worse) and management is led by a fetal medicine expert with recourse to ultrasound and computerized CTG. Another large cohort of severe early-onset FGR fetuses, classified as previable, showed a perinatal mortality of $52 \%(111 / 212$, after excluding terminations) and a diagnosis to delivery interval of 8.1 weeks for survivors ${ }^{1}$. Unfortunately, neither of these studies had information on placental biomarkers.

Various biomarkers have been proposed to aid in the identification of pregnancies at risk of an adverse pregnancy outcome although the definition of adverse outcome is often a mixture of fetal/neonatal death and morbidity related outcomes of varying severity and clinical importance. With regards to prediction of low birth weight, the most effective markers to date appears to be PIGF 16-18. A panel of angiogenic biomarkers, including PIGF and sFIt-1, taken at 24-28 weeks of gestation appears to be a good predictor of subsequent fetal demise (Relative risk of 29.1) when the biomarkers are grossly abnormal ${ }^{19}$. Furthermore, when PIGF/sFIt-1 ratio at 30-34 weeks of gestation is characterized as grossly abnormal $(<0.12 \mathrm{MoM})$ it appears to be associated with subsequent stillbirth (80\% sensitivity, $94 \%$ specificity) ${ }^{20}$. Unfortunately, the use of biomarkers to prevent perinatal mortality in RCTs has been limited with only two small studies of oestrogen and human placental lactogen included in a recent Cochrane review, neither of which showed a beneficial effect ${ }^{21}$.

In light of the promising data from small studies on biomarker prediction and the significant pathology in our STRIDER cohort, we hypothesized that a prediction model 
based upon measurable clinical features on ultrasound and biomarkers for placental disease could be valuable in providing prognostic information to women and clinicians.

\section{Methods}

This is a secondary analysis of the association between fetal biometric measurements, Doppler indices and maternal angiogenic biomarkers at the time of diagnosis of severe early-onset FGR with pregnancy outcome from the STRIDER trial ${ }^{13}$.

STRIDER was a multicenter randomised controlled trial of sildenafil vs placebo for the treatment of severe early-onset IUGR defined as a singleton pregnancy between $22^{+0}$ and $29^{+6}$ weeks of gestation with; i) a fetus with abdominal circumference (AC) or estimated fetal weight (EFW) below the $10^{\text {th }}$ centile and ii) absent or reversed end diastolic flow in the umbilical artery on Doppler velocimetry.

Following informed consent women were randomised to receive sildenafil or placebo (25 mg three times per day) until delivery or $32+0$ weeks with decision to deliver determined by the attending clinical team. Doppler, growth and blood pressure were assessed a minimum of weekly by a fetal medicine specialist. Blood samples were taken before treatment and at regular points over the next 2 weeks. There was no change in angiogenic blood parameters over time which is demonstrated by the fitting of longitudinal models and assessing the slope term (data not shown). As there was no change, only blood biomarkers from the time of diagnosis of FGR have been considered for this analysis.

Doppler ultrasound was performed serially in four vessels; the umbilical artery (UA), middle cerebral artery (MCA), ductus venosus (DV) and uterine artery (UtA). In addition to the Pulsatility Index (PI), UA end diastolic flow (EDF), DV a-wave and bilateral UtA notching were recorded. Abnormal Doppler findings were defined as 
follows; UA, raised PI, absent EDF, or reversed EDF; for MCA, low $\mathrm{PI}\left(<5^{\text {th }}\right.$ centile); for DV a-wave absent or reversed, and for UtA mean PI >1.45 or bilateral notching. Serum samples $(\geq 2 \mathrm{ml})$ were analysed retrospectively. Soluble fms-like tyrosine kinase 1 (sFlt-1) and placental growth factor PIGF (pg/ml) levels were determined using the automated Elecsys $₫$ electro-chemiluminescence immunoassay platform (Roche Cobas) and the sFIt-1:PIGF ratio was calculated. Additional analysis of PIGF was performed on baseline (pretreatment) plasma samples using the Alere ${ }^{\mathrm{TM}}$ Triage ${ }^{\circledR}$ system. Maternal serum levels of vascular endothelial growth factor (VEGF) $(\mathrm{pg} / \mathrm{ml})$ and soluble endoglin (sEng) (ng/ml) were analyzed using human Quantikine ${ }^{\circledR}$ enzyme-linked immunosorbent assays (R\&D Systems). 


\section{Statistical Methodology}

Clinical covariates at randomization included; gestational age, gestational hypertension, pre-eclampsia, preterm prelabour rupture of membranes (PPROM), EFW, blood pressure, mean arterial pressure (MAP) and parity. Doppler covariates included UA EDF, DV a-wave, MCA and UtA. Biomarker data included PIGF, sFlt-1, sEng and sFIt-1:PIGF ratio.

Analyses were carried out on five clinical outcomes; birth status (alive or stillborn), gestation at delivery, overall survival, neonatal morbidity and birth weight. Overall survival was defined as a hospital discharge of a live child (excluding stillbirths and neonatal deaths). Neonatal morbidity was defined as a liveborn fetus surviving to discharge and experiencing at least one of the following adverse outcomes: necrotising enterocolitis, retinopathy of prematurity, bronchopulmonary dysplasia requiring oxygen therapy, patent ductus arteriosus requiring medical or surgical treatment, the need for vasopressor therapy, neonatal infection, intraventricular hemorrhage within 6 weeks of delivery or a confirmed SAE as defined by the STRIDER protocol ${ }^{13}$.

Univariable analyses and multivariable analyses were carried out using a generalized linear modelling approach assuming a Gaussian family with identity link for continuous outcomes and a binomial family with logistic link for categorical outcomes. All terms included were considered as candidate covariates in the multivariable analysis. Selection of terms in multivariate models were performed using a forward stepwise approach evaluated using Akaikes Information Criterion (AIC). A forward approach was chosen over a backwards approach to avoid over parameterized models which included a large number of candidate covariates. 
Values of PIGF, sFIt-1, sEng, and sFlt-1:PIGF ratio were included as covariates measured on the log scale. Reported results are presented in terms of odds ratios (95\% Cl) for categorical data and mean (se) for continuous outcomes. Interval validation of the final model for each outcome is carried out using a bootstrap approach using measures of discrimination and calibration. Graphical summaries present the predicted model results along with all analyses are carried out using the statistical package R (Version 3).

\section{Results}

\section{Study population}

One hundred and thirty five women were recruited to the STRIDER trial between November 2014 and July 2016 from 18 fetal medicine units within the UK. The study population available for analysis was 105 (77.8\%) women (Table One); the data for 30 women from the 135 participants of the STRIDER trial were removed as angiogenic biomarker information was unavailable. 61 (58\%) women were recruited before $26^{+0}$ weeks and $44(42 \%)$ between $26^{+0}$ and $29^{+6}$ weeks of gestation. Of the 105 available patients, 70/105 (67\%) babies were born alive and 59/105 (56\%) were discharged alive. 46/70 (66\%) liveborn babies experienced neonatal morbidity. The median (IQR) gestation at delivery was $28.3,(26.9,29.7)$ weeks and the median (IQR) birth weight was $590 \mathrm{~g}(480,769)$.

\section{Modelling}

The results of univariable analysis for each of the five outcomes are included in Table Two. Considering clinical covariates, EFW and gestation at randomization were consistent univariable predictors for all outcomes. Pre-eclampsia was a univariable 
indicator of a lower gestational age at delivery (est [se] = -1.06 [0.506]; p-value =0.038) and a higher diastolic blood pressure was a univariable indicator of a greater birth weight (est [se] = 1.46 [0.699]; p-value =0.039). Reversed UA EDF Doppler (est [se] $=-1.08$ [0.417]; $p$-value $=0.011)$ was a univariable indicator of gestation at delivery. All four biomarker measures (PIGF, sEng, sFlt and sFIt-1:PIGF ratio) were univariable indicators of gestation at delivery and birth weight. PIGF and the sFIt-1:PIGF ratio were significant univariable indicators of livebirth and overall survival.

The results of univariable modelling selected are included in Table Two. The results of multivariable modelling are included in Table Three and include model intercepts for completeness. The results for each outcome are discussed separately.

Livebirths: As EFW increases, the odds of a live birth also increases [OR: 1.01 (1.008, 1.021); p-value <0.001]. Conversely a lower sFIt-1:PIGF ratio [OR: $0.53(0.284,0.994)$; $p$-value $=0.048]$ is associated with a larger probability of overall neonatal survival. The intercept of $0.14(0.003,7.39)$ allows estimation of the probability of a live birth (Figure One). For example; for a fetus with an estimated fetal weight of $400 \mathrm{~g}$ and a (log) sFlt1 :PIGF ratio of 4 , the estimated probability of a live birth is $37 \%$ as shown below.

$$
\begin{gathered}
\operatorname{logit}(p)=\log (0.14)+400 \log (1.01)+4 \log (0.53) \\
p=0.3716
\end{gathered}
$$

Gestation at birth: The presence of pre-eclampsia reduced the gestation at birth by almost a week [Est: $-0.97(-1.8,-0.2) ; p$-value $=0.020$ ] whilst having a later gestational age at randomization delayed the gestation at birth [Est: $0.61(0.5,0.7)$; p-value <0.001]. Regarding Doppler measurement, having reversed EDF in the umbilical artery [Est: $-0.97(-1.6,-0.3) p$-value $<0.001]$ resulted in an earlier gestation at delivery 
With respect to biomarker data, a higher sFIt-1:PIGF ratio [Est: -0.6 (-0.8, -0.3); p-value $<0.001]$ led to an earlier gestation at delivery.

Overall survival: As EFW increases, the odds of overall survival increase [OR: 1.01 $(1.006,1.015) ; p$-value <0.001]. A lower sFIt-1:PIGF ratio [OR: $0.51(0.286,0.904)$; $p$ value $=0.021]$ is also associated with a larger probability of overall neonatal survival. Figure Two shows the relationship between EFW and sFIt-1:PIGF ratio at diagnosis and neonatal survival. A graphical representation of the model results is also provided to give predicted probabilities of overall neonatal survival based on EFW and sFIt1:PIGF ratio (Figure Two).

Neonatal Morbidity: The only covariate chosen for inclusion in the multivariate model of neonatal morbidity was EFW. As EFW increases the probability of neonatal morbidity decreases [Est: $0.99(0.994,0.999) ; p$-value $=0.002]$.

Birth Weight: The birth weight was greater if the EFW [Est: 1.38 (1.14, 1.61); p-value $<0.001$ ] at first scan was larger. However, if the gestation at first scan was higher this had a negative impact on birth weight [Est: $-26.62(-49.64,-3.61)$; $p$-value $=0.026$ ] Considering clinical characteristics, primiparity was associated with a greater birth weight [Est: $66.36(15.67,117.05) ;$ p-value $=0.012$ ]. Considering biomarker covariates, an increased level of sEng [Est: $61.94(12.72,111.15) ; p$-value $=0.015$ ] was associated with greater birth weight whereas an increased sFIt-1:PIGF ratio [Est: $-119.43(-151.94,86.93) ; p$-value $<0.001]$ was associated with lower birth weight.

Recognising that gestational age is an important predictor of long term neonatal 
outcome, model investigations were performed replacing EFW at randomization with gestation at randomization. Comparison of the two models is given in supplementary Table One. This shows that a model including gestational age at randomization has both a larger model deviance (104.9 compared to 89.0 ) and a smaller measure of concordance (c-statistic) (0.88 compared to 0.83 ) showing better model fit for the model that includes EFW rather than gestational age at randomization. The impact of the sFIt-1:PIGF ratio did not materially change between the two models. A further summary of the internal validation used to assess the performance of each model is included in the supplementary information.

\section{DISCUSSION}

Our study has confirmed that the combination of clinical biometric data routinely recorded in cases of early-onset FGR with the addition of sFIt-1:PIGF ratio can predict pregnancy outcome for both livebirth, gestation at delivery, birthweight and overall survival. Other covariates also showed benefit in predicting gestation at delivery and birth weight. EFW was a consistent predictor for all outcomes other than gestation at delivery. sFlt-1:PIGF ratio was a consistent predictor for all outcomes other than neonatal morbidity.

To date the majority of published data have demonstrated that a low PIGF or raised sFIt-1:PIGF ratio are associated with a greater likelihood of stillbirth with very few stillbirths when results are normal ${ }^{18,22,23 ~ 23-25}$. Furthermore, an abnormal PIGF appears to more than double the risk of adverse pregnancy outcome ${ }^{26}$ and is associated with critical fetal growth restriction ${ }^{2,27-31}$ and placental pathology ${ }^{18}$. A recent Cochrane Diagnostic Test Accuracy Review on the effectiveness of biomarkers to predict 
stillbirth ${ }^{32}$ calculated that an abnormal PIGF or sFIt-1/PIGF ratio have a diagnostic odds ratio of 49.2 for subsequent stillbirth.

The strength of our model is that it combines the diagnostic sensitivity of the sFlt1:PIGF ratio with the routinely collected clinical covariates of EFW and gestational age to determine likelihood of livebirth and overall survival. We feel that the predictive model for livebirth and overall survival using EFW and sFIt-1/PIGF ratio is the most likely to be of clinical benefit to both clinicians and parents when planning the management of pregnancies affected by severe early-onset IUGR. It may aid in the planning of fetal monitoring, timing of delivery, place of birth and neonatal services. Further large cohort studies will be required to confirm the benefits of this prediction model in severe early-onset FGR and as a predictive test for adverse pregnancy outcome.

\section{Limitations}

Some covariates (PPROM, DV A-vave, UA) were not considered for inclusion in the modelling as the distribution of patients in these groups would not allow for reliable model estimates.

We were unable to assess some clinical covariates within our univariate model due to a limited number of cases with this outcome in our cohort. Further prospective sampling will be able to improve the model fit.

Interval validation has been performed but independent prospective data collection would ideally be available in another similar cohort to confirm our models findings.

\section{STRIDER study group}


Umber Agarwal, Elaine Willis and Silvia Mammarella, Liverpool Women's Hospital; Geraldine Masson, University Hospital of North Midlands, Stoke-on-Trent; Joe Aquilina, Elena Greco and Sally Higgins, The Royal London Hospital; Dimuthu Vinayagam and Louise Shaw, St. George's Hospital, London; Louise Stephens, St. Mary's Hospital, Manchester; David Howe and Abby Rand, Princess Anne Hospital, Southampton; Shalini Patni, Birmingham Heartlands Hospital; Tommy Mousa and Asma Rabab, Leicester Royal Infirmary; Helen Russell, NHS Fife Victoria Hospital, Scotland; Therese Hannon and Andrea Fenn, Royal Victoria Infirmary, Newcastle; Mark Kilby and Tara Selman, Birmingham Women's Hospital; Anna David and Rebecca Spencer, University College London Hospital; Kelly Cohen and Andrew Breeze, Leeds General Infirmary; Alastair McKelvey, Norfolk and Norwich University Hospitals, Lawrence Impey and Christos Loannou, John Radcliffe Hospital, Oxford; Sarah Stock, The Royal Infirmary of Edinburgh, Scotland; Liona Poon, Kings College London, Dharmintra Pasupathy and Louise Webster, St. Thomas' Hospital, London; and George Bugg, Nottingham City Hospital and Queen's Medical Centre.

\section{ACKNOWLEDGMENT}

We would like to thank all the women who participated in this study during such a distressing time for them and their families. We would like to thank the members of the Trial Steering Committee (Professor Alan Cameron - Chair, Professor Elizabeth Draper, Dr Paul Clarke, Dr Laura Price, Dr Laura Bonnett, Mr Alex Astor, Ms Louise Hardman, and Miss Karen Wilding), Independent Safety and Data Monitoring Committee (Professor Ed Juszczak - Chair, Professor Christoph Lees and Dr Ben Stenson) and all the individuals who helped with the management and conduct of the STRIDER UK study. We are also grateful to Sharp Clinical Services and University of 
British Columbia (UBC), Canada for supporting the provision of blinded IMP to research sites and to staff of pharmacy and research and development departments in all of the participating hospitals. We would also like to thank UBC for the development and support of the STRIDER randomization and electronic data capture systems and Liverpool Clinical Laboratories, Royal Liverpool University and Broadgreen Hospital Trust for the performance of Elecsys ${ }^{\circledR}$ sFlt-1 and PIGF (Roche Cobas) analyses.

\section{FUNDING}

STRIDER was funded by the Efficacy and Mechanism Evaluation (EME) Programme, a Medical Research Council (MRC) and National Institute of health research (NIHR) partnership, award number 12/62/109. The EME Programme is funded by the MRC and NIHR, with contributions from the Chief Scientist Office in Scotland and National Institute for Social Care and Research in Wales.

\section{DISCLAIMER}

This report is independent research funded by the MRC and managed by the NIHR on behalf of the MRC-NIHR partnership. The views expressed in this publication are those of the authors and not necessarily those of the MRC, NHS, NIHR or the Department of Health.

\section{References}

1. Lawin-O'Brien AR, Dall'Asta A, Knight C, et al. Short-term outcome of periviable small-forgestational-age babies: is our counseling up to date? Ultrasound Obstet Gynecol 2016;48:636-41.

2. Baschat AA, Cosmi E, Bilardo CM, et al. Predictors of neonatal outcome in early-onset placental dysfunction. Obstet Gynecol 2007;109:253-61.

3. Temming LA, Dicke JM, Stout MJ, et al. Early Second-Trimester Fetal Growth Restriction and Adverse Perinatal Outcomes. Obstet Gynecol 2017;130:865-9. 
4. Lundgren E, Cnattingius S, Jonsson B, Tuvermo T. Intellectual and psychological perfromance in males born small for gestational age with and without catch-up growth. Pediatr Res 2001;50:91-6. 5. Bardin C, Piuze G, Papageorgiou A. Outcome at 5 years of age of SGA and AGA infants born less than 28 weeks of gestation. Semin Perinatol 2004;28:288-94.

6. Sung I, Vohr B, Oh W. Growth and neurodevelopmental outcome of very low birth weight infants with intrauterine growth retardation: comparison with control subjects matched by birth weight and gestational age. J Pediatr 1993;123:618-24.

7. Lees CC, Marlow N, van Wassenaer-Leemhuis A, et al. 2 year neurodevelopmental and intermediate perinatal outcomes in infants with very preterm fetal growth restriction (TRUFFLE): a randomised trial. Lancet 2015;385:2162-72.

8. Lienhardt A, carel J, Preux P, Coutant R, Chaussain J. Amplitude of pubertal growth in short stature children with intrauterine growth retardation. Horm Res 2002;57:88-94.

9. Stein C, Fall C, Kumaran K, Osmond C, Cox V, Barker D. Fetal growth and coronary heart disease in south india. Lancet 1996;348:1269-73.

10. Barker DJ. The developmental origins of chronic adult disease. Acta Paediatr Suppl 2004;93:26-33.

11. Lees C, Marlow N, Arabin B, et al. Perinatal morbidity and mortality in early-onset fetal growth restriction: cohort outcomes of the trial of randomised umbilical and fetal flow in Europe (TRUFFLE). Ultrasound Obstet Gynecol 2013;42:400-8.

12. Lin CS, Lin G, Xin ZC, Lue TF. Expression, distribution and regulation of phosphodiesterase 5 . Curr Pharm Des 2006;12:3439-57.

13. Sharp A, Cornforth C, Jackson R, et al. Maternal sildenafil for severe fetal growth restriction (STRIDER): a multicentre, randomised, placebo-controlled, double-blind trial. The Lancet Child \& Adolescent Health 2018.

14. Sharp AN, Alfirevic Z. First trimester screening can predict adverse pregnancy outcomes. Prenat Diagn 2014;34:660-7.

15. Sharp AN, Heazell AE, Crocker IP, Mor G. Placental apoptosis in health and disease. Am J Reprod Immunol 2010;64:159-69.

16. Griffin M, Seed PT, Duckworth S, et al. Predicting delivery of a small-for-gestational-age infant and adverse perinatal outcome in women with suspected pre-eclampsia. Ultrasound Obstet Gynecol 2018;51:387-95.

17. Dutton PJ, Warrander LK, Roberts SA, et al. Predictors of poor perinatal outcome following maternal perception of reduced fetal movements--a prospective cohort study. PLoS One 2012;7:e39784.

18. Benton SJ, McCowan LM, Heazell AE, et al. Placental growth factor as a marker of fetal growth restriction caused by placental dysfunction. Placenta 2016;42:1-8.

19. Chaiworapongsa $\mathrm{T}$, Romero $\mathrm{R}$, Erez $\mathrm{O}$, et al. The prediction of fetal death with a simple maternal blood test at 20-24 weeks: a role for angiogenic index-1 (PIGF/sVEGFR-1 ratio). Am J Obstet Gynecol 2017;217:682 e1- e13.

20. Chaiworapongsa T, Romero R, Korzeniewski SJ, et al. Maternal plasma concentrations of angiogenic/antiangiogenic factors in the third trimester of pregnancy to identify the patient at risk for stillbirth at or near term and severe late preeclampsia. Am J Obstet Gynecol 2013;208:287 e1e15.

21. Heazell AE, Whitworth M, Duley L, Thornton JG. Use of biochemical tests of placental function for improving pregnancy outcome. Cochrane Database Syst Rev 2015:CD011202.

22. Chappell L, Duckworth S, Seed P, et al. Diagnostic accuracy of placental growth factor in women with suspected preeclampia: a prospective multicenter study. Circulation 2013;128:2121-31.

23. Navaratnam K, Abreu P, Clarke H, Jorgensen A, Alfirevic A, Alfirevic Z. Evaluation of agreement of placental growth factor (PIGF) tests and the soluble FMS-like tyrosine kinase 1 (sFlt1)/PIGF ratio, comparison of predictive accuracy for pre-eclampsia, and relation to uterine artery Doppler and response to aspirin. J Matern Fetal Neonatal Med 2017:1-9. 
24. Chaiworapongsa T, Romero R, Erez O, et al. A low angiogenic index-1 (PIGF/sVEGFR-1 ratio) at 24-28 weeks of gestation is a biomarker to identify the patient at risk for subsequent fetal death. Am J Obstet Gynecol 2017.

25. Zeisler H, Llurba E, Chantraine F, et al. Predictive Value of the sFlt-1:PIGF Ratio in Women with Suspected Preeclampsia. N Engl J Med 2016;374:13-22.

26. Miranda J, Triunfo S, Rodriguez-Lopez $\mathrm{M}$, et al. Performance of third-trimester combined screening model for prediction of adverse perinatal outcome. Ultrasound Obstet Gynecol 2017;50:353-60.

27. Crimmins S, Desai A, Block-Abraham D, Berg C, Gembruch U, Baschat AA. A comparison of Doppler and biophysical findings between liveborn and stillborn growth-restricted fetuses. Am J Obstet Gynecol 2014;211:669 e1- e10.

28. Figueras F, Gratacos E. Stage-based approach to the management of fetal growth restriction. Prenat Diagn 2014;34:655-9.

29. Cruz-Martinez R, Figueras F, Hernandez-Andrade E, Oros D, Gratacos E. Fetal brain Doppler to predict cesarean delivery for nonreassuring fetal status in term small-for-gestational-age fetuses. Obstet Gynecol 2011;117:618-26.

30. Hershkovitz R, Kingdom JC, Geary M, Rodeck CH. Fetal cerebral blood flow redistribution in late gestation: identification of compromise in small fetuses with normal umbilical artery Doppler. Ultrasound Obstet Gynecol 2000;15:209-12.

31. Flood K, Unterscheider J, Daly S, et al. The role of brain sparing in the prediction of adverse outcomes in intrauterine growth restriction: results of the multicenter PORTO Study. Am J Obstet Gynecol 2014;211:288 e1-5.

32. Heazell AE, Hayes DJL, Whitworth M, Takwoingi Y, Bayliss SE, C. D. Diagnostic accuaracy of biochemical tests of placental function versus ultrasound assessment of fetal size for stillbirth and small-for-gestational-age infants (Protocol). Cochrane Database Syst Rev 2016;No.: CD012245.

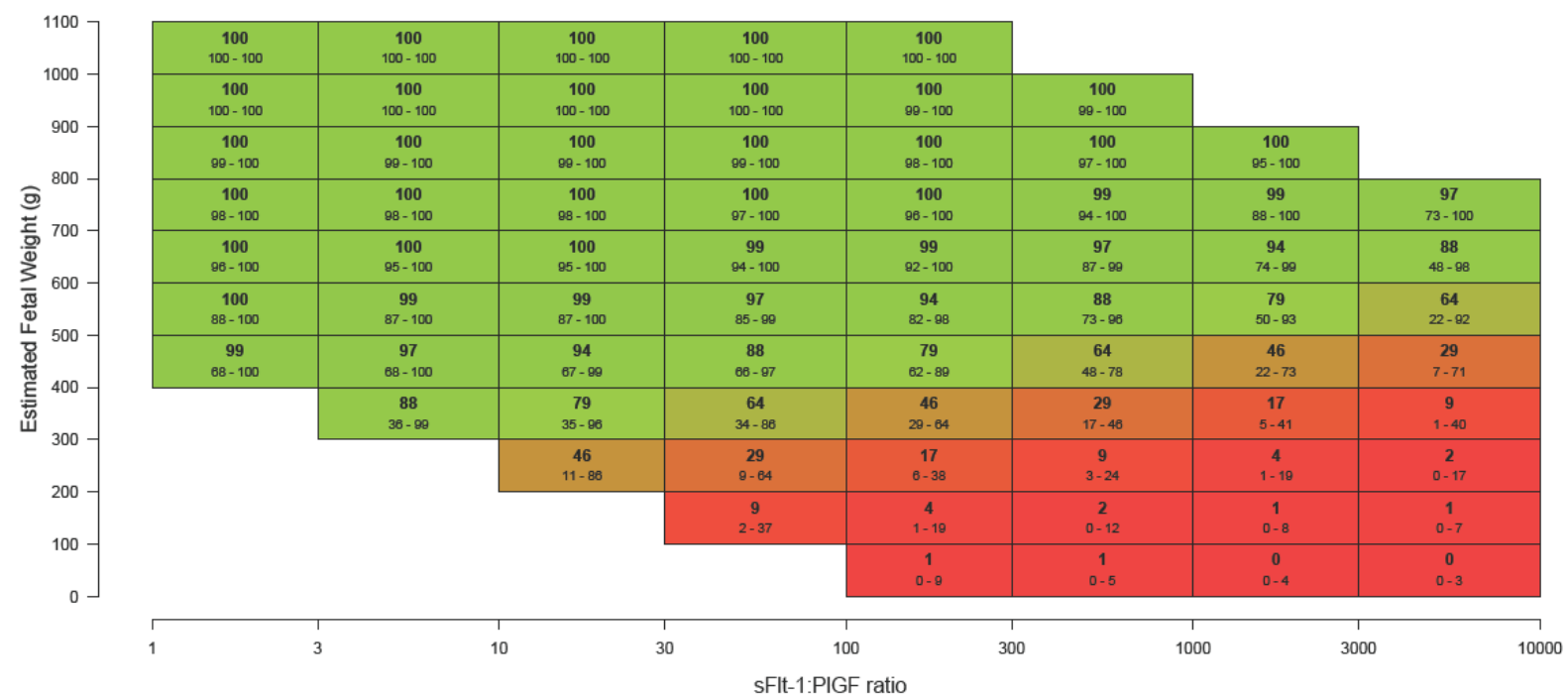




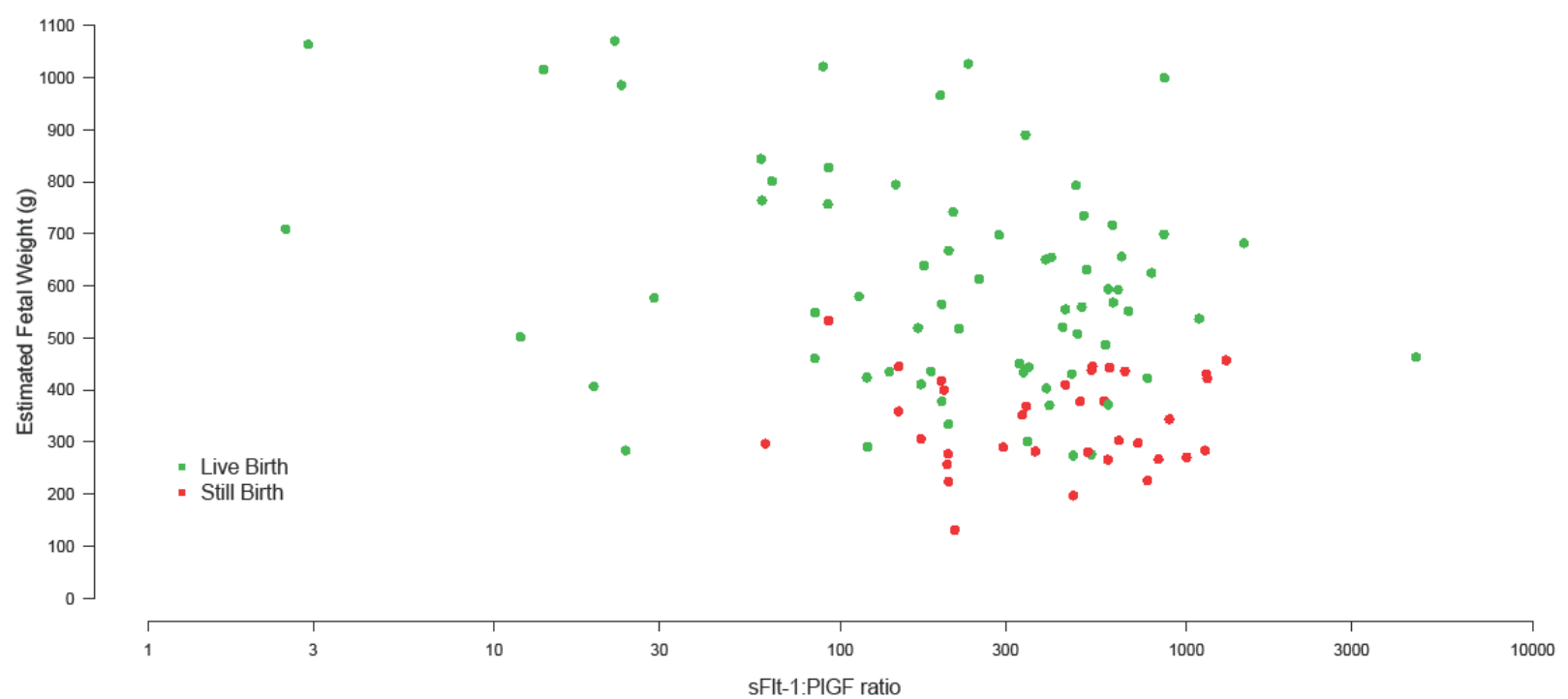

Figure 1: Predicted probability of Livebirth based on both the estimated fetal weight and the sFIt-1:PIGF ratio

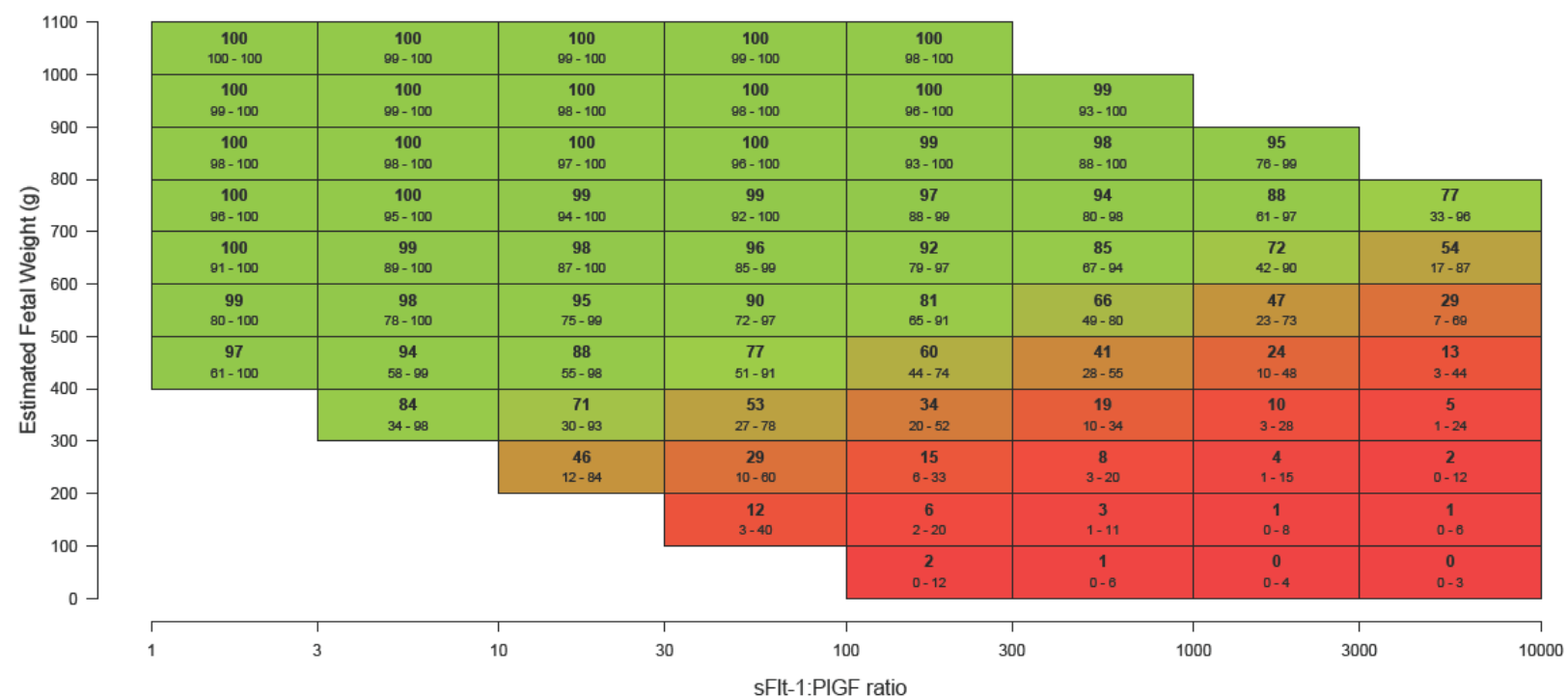




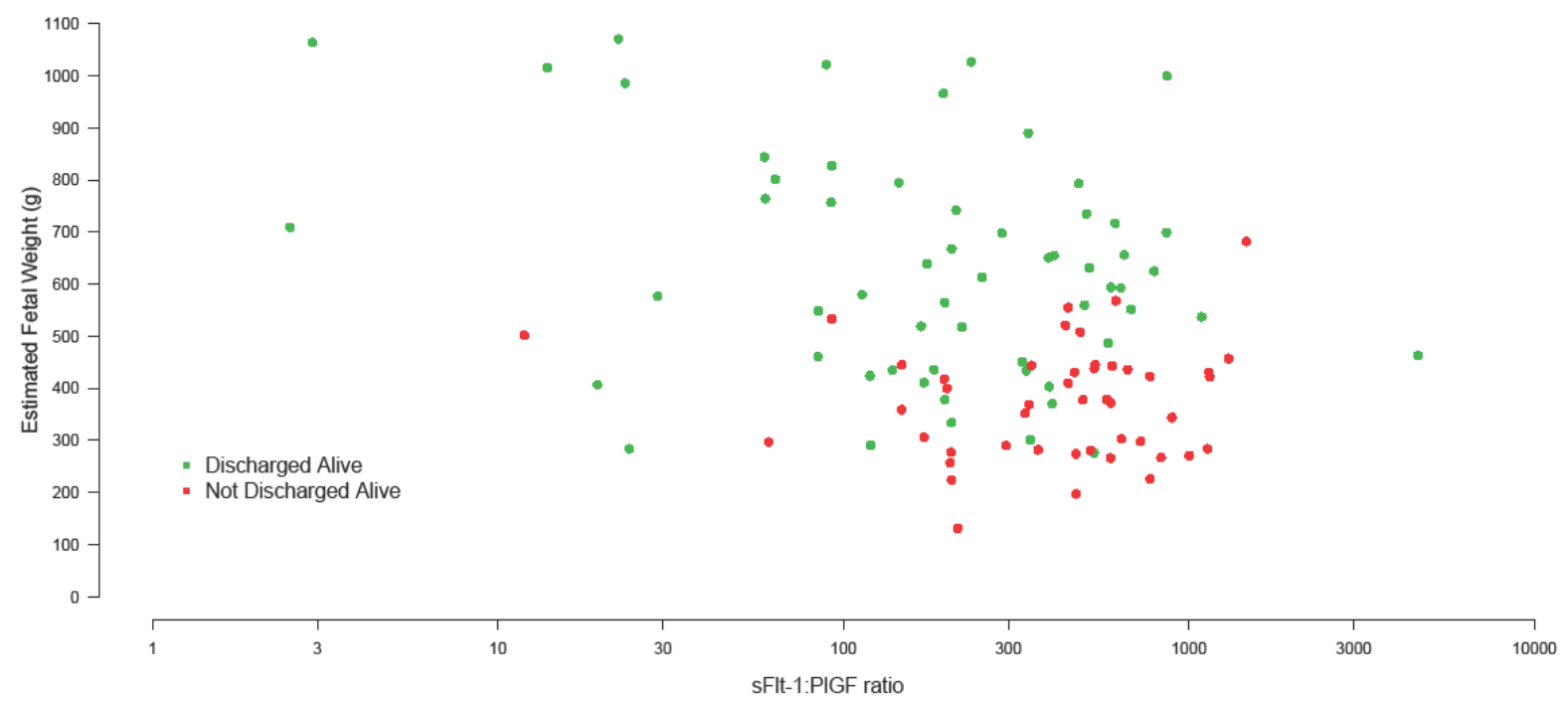

Figure 2: Predicted probability of overall survival based on both the estimated fetal weight and the sFIt-1:PIGF ratio 
1.1 Table 1: Patient Demographics

\begin{tabular}{|c|c|c|}
\hline Covariate & Level & $\begin{array}{c}\text { Total } \\
\mathrm{n}=105\end{array}$ \\
\hline Age & Median (IQR) & $30(27,35)$ \\
\hline \multirow{2}{*}{ Previous Pregnancy } & No & 54 \\
\hline & Yes & 51 \\
\hline \multirow{2}{*}{ Gestational Hypertension } & No & 81 \\
\hline & Yes & 24 \\
\hline \multirow{2}{*}{ Pre-Eclampsia } & No & 87 \\
\hline & Yes & 18 \\
\hline Diastolic Blood Pressure & Median (IQR) & $88.5(79.5,95.5)$ \\
\hline Systolic Blood Pressure & Median (IQR) & $134.5(124.5,144.5)$ \\
\hline Mean Arterial Pressure & Median (IQR) & $104.167(93.5,111.667)$ \\
\hline \multirow{2}{*}{ PPROM } & No & 104 \\
\hline & Yes & 1 \\
\hline Estimated Fetal Weight & Median (IQR) & $451(369,651)$ \\
\hline Gestation at Randomization & Median (IQR) & $25.286(24.143,27.429)$ \\
\hline \multirow{2}{*}{ EDF } & Absent & 74 \\
\hline & Reversed & 29 \\
\hline \multirow{2}{*}{ DV a-wave } & A wave Positive & 93 \\
\hline & A wave Reversed & 7 \\
\hline \multirow{2}{*}{ MCA } & Abnormal & 53 \\
\hline & Normal & 42 \\
\hline \multirow{3}{*}{ UA } & No Notch $(<1.45)$ & 13 \\
\hline & No Notch $(\geq 1.45)$ & 7 \\
\hline & Notch & 73 \\
\hline \multirow{2}{*}{ Birth Status } & Stillbirth & 35 \\
\hline & Livebirth & 70 \\
\hline Gestation at Delivery & Median (IQR) & $28.286(26.857,29.714)$ \\
\hline \multirow{2}{*}{ Neonatal Outcome } & Died (SB/NND) & 46 \\
\hline & Survived & 59 \\
\hline \multirow{2}{*}{ Neonatal Morbidity } & No & 24 \\
\hline & Yes & 46 \\
\hline Birthweight & Median (IQR) & $590(480,769)$ \\
\hline PIGF & Median (IQR) & $22(13,42)$ \\
\hline sEng & Median (IQR) & $33.61(20.36,57.82)$ \\
\hline sFlt-1 & Median (IQR) & $6894(5025,10380)$ \\
\hline sFIt-1/PIGF & Median (IQR) & $343.9(167.2,591.6)$ \\
\hline
\end{tabular}

Table 1: Patient Demographics for the whole Strider Cohort 
1.1 TABLE 2: UNIVARIATE ANALYSIS

\begin{tabular}{|c|c|c|c|c|c|c|c|c|c|c|c|}
\hline \multirow{2}{*}{ Covariate } & \multirow{2}{*}{ Level } & \multicolumn{2}{|l|}{ Livebirth } & \multicolumn{2}{|c|}{ Gestation at Birth } & \multicolumn{2}{|c|}{ Overall Survival } & \multicolumn{2}{|c|}{ Neonatal Morbidity } & \multicolumn{2}{|c|}{ Birth Weight } \\
\hline & & Est & $P$ & Est & $\mathrm{P}$ & Est & $P$ & Est & $\mathrm{P}$ & Est & $P$ \\
\hline \multirow{2}{*}{ Allocation } & Placebo & & & & & & & & & & \\
\hline & Sildenafil & $1.19(0.578,2.464)$ & 0.632 & $-0.42(0.392)$ & 0.285 & $1.01(0.514,1.999)$ & 0.969 & $1.81(0.75,4.369)$ & 0.187 & $-13.41(56.726)$ & 0.813 \\
\hline Age & & $0.97(0.911,1.039)$ & 0.411 & $-0.02(0.035)$ & 0.615 & $0.98(0.925,1.045)$ & 0.587 & $1.01(0.932,1.089)$ & 0.852 & 0.28 (5.101) & 0.956 \\
\hline \multirow{2}{*}{ Previous pregnancy } & No & & & & & & & & & & \\
\hline & Yes & $1.02(0.49,2.105)$ & 0.967 & $0.29(0.395)$ & 0.471 & $0.85(0.43,1.684)$ & 0.642 & $1.32(0.544,3.193)$ & 0.54 & 71.91 (56.71) & 0.207 \\
\hline \multirow{2}{*}{$\begin{array}{l}\text { Gestational } \\
\text { Hypertension }\end{array}$} & No & & & & & & & & & & \\
\hline & Yes & $1.23(0.53,2.862)$ & 0.629 & $-0.22(0.449)$ & 0.623 & $0.93(0.431,2.022)$ & 0.861 & $0.64(0.245,1.661)$ & 0.357 & $1.56(64.691)$ & 0.981 \\
\hline \multirow{2}{*}{ Pre-Eclampsia } & No & & & & & & & & & & \\
\hline & Yes & $1.5(0.549,4.097)$ & 0.429 & $-1.05(0.505)$ & 0.038 & $0.93(0.385,2.266)$ & 0.88 & $2.87(0.76,10.827)$ & 0.119 & $-73.44(73.876)$ & 0.322 \\
\hline $\begin{array}{l}\text { Diastolic Blood } \\
\text { Pressure } \\
\end{array}$ & & $1.01(0.984,1.032)$ & 0.532 & $0.01(0.005)$ & 0.142 & $1.01(0.988,1.025)$ & 0.471 & $0.98(0.942,1.019)$ & 0.298 & $1.46(0.699)$ & 0.039 \\
\hline $\begin{array}{l}\text { Systolic Blood } \\
\text { Pressure }\end{array}$ & & $1(0.973,1.019)$ & 0.711 & $-0.02(0.012)$ & 0.079 & $0.99(0.969,1.012)$ & 0.394 & $\begin{array}{c}1(0.972 P \text { IGF and } \mathrm{t} \\
1.033) \\
\end{array}$ & 0.897 & $-1.54(1.801)$ & 0.395 \\
\hline Mean Arterial Pressure & & $1.01(0.987,1.026)$ & 0.54 & $0.01(0.007)$ & 0.288 & $1.01(0.99,1.021)$ & 0.513 & $0.99(0.958,1.013)$ & 0.299 & $1.81(1.00)$ & 0.072 \\
\hline $\begin{array}{l}\text { Estimated Fetal } \\
\text { Weight }\end{array}$ & & $1.02(1.01,1.022)$ & $<0.001$ & $0.01(0.001)$ & $<0.001$ & $1.01(1.007,1.015)$ & $\varangle 0.001$ & $1(0.994,0.999)$ & 0.002 & $1.31(0.066)$ & $<0.001$ \\
\hline $\begin{array}{l}\text { Gestabion at } \\
\text { Randomization }\end{array}$ & & $2.16(1.623,2.874)$ & $<0.001$ & $0.68(0.067)$ & $<0.001$ & $2(1.566,2.542)$ & $<0.001$ & $0.63(0.485,0.824)$ & 0.001 & $103.23(9.276)$ & $<0.001$ \\
\hline \multirow{2}{*}{ EDF } & Absent & & & & & & & & & & \\
\hline & Reversed & $0.8(0.37,1.732)$ & 0.571 & $-1.08(0.417)$ & 0.011 & $0.65(0.313,1.359)$ & 0.254 & $1.64(0.603,4.475)$ & 0.331 & $-57.29(61.465)$ & 0.353 \\
\hline \multirow{2}{*}{ MCA } & Abnormal & & & & & & & & & & \\
\hline & Normal & $1.42(0.653,3.081)$ & 0.377 & $0.56(0.427)$ & 0.192 & $1.05(0.511,2.148)$ & 0.9 & $0.93(0.372,2.341)$ & 0.883 & 28.44 (61.676) & 0.646 \\
\hline \multirow{3}{*}{ UA } & No Notch $(<1.45)$ & & & & & & & & & & \\
\hline & No Notch (21.45) & $0.43(0.08,2.308)$ & 0.324 & $0.84(0.138,5.07)$ & 0.847 & $0.64(0.134,3.029)$ & 0.57 & $0.28(0.037,2.092)$ & 0.214 & $-16.7(129.571)$ & 0.898 \\
\hline & Notch & $0.48(0.145,1.574)$ & 0.225 & $0.33(0.102,1.079)$ & 0.069 & $0.62(0.222,1.749)$ & 0.368 & $1.42(0.413,4.902)$ & 0.576 & -181.21 (84.745) & 0.035 \\
\hline PIGF & & $3.89(1.9,7.956)$ & $<0.001$ & $1.4(0.219)$ & $<0.001$ & $3.67(1.903,7.058)$ & 40.001 & $0.59(0.336,1.04)$ & 0.068 & 262.6 (28.438) & $<0.001$ \\
\hline sEng & & $0.77(0.455,1.303)$ & 0.331 & $-0.67(0.274)$ & 0.015 & $0.63(0.381,1.045)$ & 0.073 & $1.22(0.672,2.227)$ & 0.509 & $-115.58(40.862)$ & 0.006 \\
\hline sflt-1 & & $1.12(0.611,2.036)$ & 0.722 & $-0.95(0.329)$ & 0.005 & $0.88(0.49,1.564)$ & 0.653 & $1.53(0.769,3.046)$ & 0.226 & $-124.97(49.403)$ & 0.013 \\
\hline sFlt-1/PIGF & & $0.56(0.355,0.872)$ & 0.011 & $-0.99(0.157)$ & $<0.001$ & $0.51(0.327,0.782)$ & 0.002 & $1.42(0.978,2.075)$ & 0.065 & $-169.72(21.821)$ & $<0.001$ \\
\hline
\end{tabular}

Table 2: Clinical covariates included for univariate analysis

\begin{tabular}{|c|c|c|c|c|c|c|c|c|c|c|c|}
\hline \multirow[b]{2}{*}{ Covariate } & \multirow[b]{2}{*}{ Level } & \multicolumn{2}{|c|}{ Livebirth } & \multicolumn{2}{|c|}{$\begin{array}{c}\text { Gestation at } \\
\text { Birth }\end{array}$} & \multicolumn{2}{|c|}{$\begin{array}{l}\text { Overall } \\
\text { Survival }\end{array}$} & \multicolumn{2}{|c|}{$\begin{array}{l}\text { Neonatal } \\
\text { Morbidity }\end{array}$} & \multicolumn{2}{|c|}{ Birth Weight } \\
\hline & & $\begin{array}{c}\text { OR } \\
(95 \\
\% \\
\mathrm{Cl})\end{array}$ & $P$ & $\begin{array}{c}\text { Est } \\
(95 \\
\% \\
\mathrm{Cl})\end{array}$ & $P$ & $\begin{array}{c}\text { OR } \\
(95 \% \\
\mathrm{Cl})\end{array}$ & $P$ & $\begin{array}{c}\text { OR } \\
(95 \\
\% \\
\mathrm{Cl})\end{array}$ & $P$ & $\begin{array}{c}\text { Est } \\
(95 \% \\
\mathrm{Cl})\end{array}$ & $P$ \\
\hline Intercept & & $\begin{array}{c}0.14 \\
(0.003 \\
, 7.39) \\
\end{array}$ & 0.33 & $\begin{array}{l}16.67 \\
(12.5 \\
20.9)\end{array}$ & $\begin{array}{c}<0.00 \\
1\end{array}$ & $\begin{array}{c}0.43 \\
(0.013, \\
14.798 \\
)\end{array}$ & 0.641 & $\begin{array}{c}21.72 \\
(4.275 \\
110.3) \\
\end{array}$ & $\begin{array}{c}<0.00 \\
1\end{array}$ & $\begin{array}{c}1050.38 \\
(544.26 \\
1556.51 \\
\quad)\end{array}$ & $\begin{array}{c}<0.00 \\
1\end{array}$ \\
\hline \multirow[b]{2}{*}{ Pre-Eclampsia } & No & & & & & & & & & & \\
\hline & Yes & & & $\begin{array}{c}-0.97 \\
(-1.8,- \\
0.2) \\
\end{array}$ & 0.020 & & & & & & \\
\hline \multirow[b]{2}{*}{ EDF } & Absent & & & & & & & & & & \\
\hline & $\begin{array}{l}\text { Reverse } \\
\mathrm{d}\end{array}$ & & & $\begin{array}{c}-0.97 \\
(-1.6,- \\
0.3) \\
\end{array}$ & $\begin{array}{c}<0.00 \\
1\end{array}$ & & & & & & \\
\hline \multirow[t]{2}{*}{$\begin{array}{l}\text { Estimated } \\
\text { Fetal Weight }\end{array}$} & $\begin{array}{l}\text { median } \\
\text { (IQR) }\end{array}$ & $\begin{array}{c}1.01 \\
(1.008 \\
1.021) \\
\end{array}$ & $\begin{array}{c}<0.00 \\
1\end{array}$ & & & $\begin{array}{c}1.01 \\
(1.006, \\
1.015)\end{array}$ & $\begin{array}{c}<0.00 \\
1\end{array}$ & $\begin{array}{c}0.99 \\
(0.994 \\
0.999) \\
\end{array}$ & 0.002 & $\begin{array}{l}1.38 \\
(1.14 \\
1.61) \\
\end{array}$ & $\begin{array}{c}<0.00 \\
1\end{array}$ \\
\hline & Yes & & & & & & & & & & \\
\hline
\end{tabular}




\begin{tabular}{|c|c|c|c|c|c|c|c|c|c|}
\hline $\begin{array}{l}\text { Previous } \\
\text { Pregnancy }\end{array}$ & No & & & & & & & $\begin{array}{c}66.36 \\
(15.67, \\
117.05)\end{array}$ & 0.012 \\
\hline $\begin{array}{l}\text { Gestation at } \\
\text { Randomizatio } \\
n\end{array}$ & $\begin{array}{l}\text { median } \\
\text { (IQR) }\end{array}$ & & & $\begin{array}{l}0.61 \\
(0.5 \\
0.7)\end{array}$ & $\begin{array}{c}<0.00 \\
1\end{array}$ & & & $\begin{array}{c}-26.62(- \\
49.64,- \\
3.61)\end{array}$ & 0.026 \\
\hline sEng & $\begin{array}{l}\text { median } \\
(\mathrm{IQR})\end{array}$ & & & & & & & $\begin{array}{l}61.94 \\
(12.72, \\
111.15)\end{array}$ & 0.015 \\
\hline sFlt-1/PIGF & $\begin{array}{l}\text { median } \\
\text { (IQR) }\end{array}$ & $\begin{array}{c}0.53 \\
(0.284 \\
\quad \\
0.994)\end{array}$ & 0.048 & $\begin{array}{c}-0.6(- \\
0.8,- \\
0.3)\end{array}$ & $\begin{array}{c}<0.00 \\
1\end{array}$ & $\begin{array}{c}0.51 \\
(0.286 \\
0.904)\end{array}$ & 0.021 & $\begin{array}{c}-119.43 \\
(- \\
151.94 \\
86.93)\end{array}$ & $\begin{array}{c}<0.00 \\
1\end{array}$ \\
\hline
\end{tabular}

Table 3: Multivariate analysis of significant covariates for main outcome measures 